\title{
Estratégia lúdica no ensino de boas práticas de higiene à crianças hospitalizadas
}

\author{
Ludic strategy in teaching good hygiene practices to \\ hospitalized children
}

\author{
Cinthya Lorena Bezerra Sarmanho', Kéven Lorena de Paula Gonçalves', \\ Maicon de Araujo Nogueira', Michele de Freitas Melo', Renato da Costa Teixeira' \\ 'Programa de Pós-graduação em Ensino e Saúde na Amazônia, Universidade do Estado do Pará, Belém (PA), \\ Brasil.
}

\section{RESUMO}

Contextualização: O presente estudo relata a experiência do uso de estratégias lúdicas no processo de ensino-aprendizagem de boas práticas de higiene corporal voltado para crianças e seus familiares, que envolveu discentes, docentes, equipe assistencial, usuários e seus cuidadores, durante o estágio supervisionado do componente curricular Enfermagem em Pediatria do Curso de Graduação em Enfermagem da Faculdade Metropolitana da Amazônia (FAMAZ), num hospital de referência em trauma de Ananindeua, Estado do Pará. Objetivou-se com o presente estudo apresentar as experiências e as atividades vivenciadas por acadêmicos de Enfermagem na orientação sobre higiene corporal voltado para crianças hospitalizadas e seus cuidadores. Descrição da experiência: Participaram do estudo todas as crianças hospitalizadas, num quantitativo de dezenove crianças e seus respectivos cuidadores, totalizando 38 participantes. A problematização foi a metodologia selecionada para o planejamento das atividades. Durante a fase de observação da realidade, os discentes foram instigados pelo professor, partindo de um tema gerador "Hábitos saudáveis e qualidade de vida", a identificarem as necessidades das crianças hospitalizadas e de seus cuidadores com vistas a desenvolverem uma ação educativa. Realizou-se uma ação educativa em saúde sobre higiene corporal, com foco na higienização das mãos, corte e limpeza das unhas, banho e escovação dental, tendo como objetivo incorporar esses hábitos não só no ambiente hospitalar, mas na vida cotidiana dos indivíduos. Considerações finais: A experiência proporcionou a todos os atores envolvidos a constatação da importância das ações de Educação em Saúde, que devem ser executadas nos mais diversos cenários, inclusive no ambiente hospitalar e sempre pautadas nas reais necessidades da população-alvo, a fim de provocar mudanças significativas nos hábitos de vida, promovendo saúde.

PALAVRAs-Chave: Educação em saúde. Higiene. Criança hospitalizada. Cuidadores.

\section{ABSTRACT}

Contextualization: This study reports the experience of the use of play strategies in the teaching-learning process for good body-hygiene practices aimed to children and their families, involving students, teachers, care staff, users and their caregivers during supervised training of the curricular component: Nursing in Pediatrics of the Undergraduate Nursing Course of the Amazon Metropolitan College (FAMAZ), in a well-known and highly regarded trauma hospital in Ananindeua, in the State of Pará. The objective of this study was to present the experiences and activities of nursing students as part of orientation on body hygiene for hospitalized children and their caregivers. Description of the experience: All the hospitalized children participated in the study, a total of 38 participants - this being nineteen children and their respective caregivers. The question to be solved were selecting the methodology to plan the activities. During the observation phase of the real situation, the students were instigated by the teacher, starting with the theme "Healthy Habits and Quality of Life", to identify the needs of hospitalized children and their caregivers, with a view to developing an educational initiative. An educational health initiative was implemented for body hygiene, focusing on hand hygiene, nail cutting and cleaning, bathing and dental brushing, with the purpose of incorporating these habits not only within the hospital environment, but also in the daily life of the individuals concerned. Final considerations: The experience provided to all the protagonists involved confirmation of the importance of the Health Education initiatives, which must be carried out in the most diverse scenarios, including in the hospital environment and always based on the real needs of the target population, in order to bring about significant changes in living habits, promoting a healthy living environment.

KEYWORDS: Health education. Hygiene. Hospitalized child. Caregivers. 


\section{Contextualização}

O presente estudo relata a experiência do uso de estratégias lúdicas no processo de ensino-aprendizagem de boas práticas de higiene corporal voltado para crianças e seus familiares, que envolveu discentes, docentes, equipe assistencial, usuários e seus cuidadores, durante o estágio supervisionado do componente curricular "Assistência de Enfermagem em Pediatria" do Curso de Graduação em Enfermagem da Faculdade Metropolitana da Amazônia (FAMAZ), num hospital de referência em trauma de Ananindeua, Estado do Pará. Nesse contexto, foi possível estabelecer um contato mais próximo com crianças hospitalizadas e seus cuidadores, que na sua maioria estavam na companhia de pais/familiares.

As crianças representam um segmento de relevância na sociedade, tanto pela necessidade de serem cuidadas por adultos como pelo impacto que agravos e hábitos, ocorridos ou iniciados nessa etapa, podem exercer em sua saúde ao longo da vida. Além disso, na infância ocorrem várias modificações, particularmente físicas e psicológicas, que caracterizam o crescimento e o desenvolvimento da criança ${ }^{1}$.

A hospitalização de crianças constitui um evento em que há necessidade de maior comunicação, informação adequada e negociação de cuidados, havendo barreiras e facilidades para a participação de cuidadores. A participação dos pais nos cuidados de saúde de crianças hospitalizadas é tema que vem sendo pesquisado, destacando o hospital como um ambiente não familiar e que traz mudanças nos papéis familiares. Os conhecimentos acerca das necessidades dos cuidadores durante o acompanhamento da criança doente, em contexto hospitalar, permitem salientar o interesse e envolvimento nos cuidados de saúde em pediatria, de modo a contribuir para a atenção à saúde qualificada e humanizada².

A hospitalização é uma situação crítica e delicada na vida de qualquer ser humano, mas na vida de uma criança adquire características especiais, pois implica na mudança de rotina de toda a família. Neste período, é comum o afastamento da escola, dos amigos e do domicílio. Os hábitos da criança e do familiar cuidador ficam dependentes da rotina da unidade hospitalar, o que pode gerar-lhes ansiedade; além disso, por vezes os familiares perdem a autonomia de sua vida ${ }^{3}$.

$\mathrm{Na}$ assistência à criança, o cuidado deve contemplar não somente os aspectos técnicos, como também as necessidades físicas, emocionais e sociais, visando ao impacto positivo na vida pessoal e familiar. A experiência de sofrimento pode ser exacerbada com a internação da criança, gerando incertezas e sobrecarga. Assim, destaca-se o papel essencial dos profissionais de saúde junto ao binômio cuidador-criança doente, como provedores de cuidado integral, de qualidade e mais humano, acolhendo-os da melhor forma possível, por meio de relação ética, proporcionando a formação de vínculos terapêuticos ${ }^{4}$.

No contexto da internação pediátrica é relevante encontrar espaços para a educação em saúde, a qual deve permear todas as práticas do cuidado infantil e envolver os familiares nessas ações. Assim, tem-se no ambiente hospitalar a oportunidade de desenvolver questões de educação em saúde com os familiares cuidadores inseridos neste espaço, mediante o desenvolvimento de ações que possibilitem a educação em saúde a partir da situação de saúde/doença da criança, para a promoção de saúde da família ${ }^{3}$.

Ante a essa realidade, as ações educativas representam estratégias de apoio, aprendizado, independência e motivação para autogerenciamento do cuidado, por meio de ações dialógicas e emancipatórias ${ }^{4}$.

Para alcançar uma comunicação mais efetiva é indispensável que os profissionais de saúde conheçam a realidade, a visão de mundo e as expectativas de cada sujeito, para que possam priorizar as necessidades dos indivíduos e não apenas as exigências terapêuticas. Isso se torna possível a partir do conhecimento preexistente, pois o não reconhecimento desses aspectos pode desvalorizar experiências, e desencadear uma 
série de consequências, como a não adesão ao tratamento, descrédito em relação à terapêutica, deficiência no autocuidado, adoção de crenças e hábitos prejudiciais à saúde, distanciamento da equipe multiprofissional ${ }^{4}$.

O potencial da Educação em Saúde deve ser reconhecido como abordagem integral que inclui a prevenção de agravos e a promoção e prevenção da saúde, por meio de ações que possam estimular a participação da população e que permita aproximação ao contexto de vida das pessoas. Nesta perspectiva, crianças e famílias são capazes de apreender e compartilhar conhecimentos sobre qualidade de vida e saúde. As reflexões e os questionamentos sobre o enfoque educativo emergiram ao reconhecer que, frequentemente, na prática assistencial, o Enfermeiro se afasta desta atividade, ou não a desenvolve, com vistas a contemplar as necessidades e condições de aprendizagem da criança, de modo que ela e a família possam entender, decidir e agir sobre seus determinantes de saúde, aderindo aos projetos terapêuticos compartilhados com a equipe que se expressem para além da hospitalização.

Diante do exposto emergiram os seguintes questionamentos: como o acadêmico de enfermagem percebe a prática educativa no cuidado de crianças hospitalizadas? Qual o impacto que ações educativas dinamizadoras, e focadas no individuo podem proporcionar? Vislumbra-se a possibilidade de colaborar com reflexões que despertem o desenvolvimento de atividades educativas junto às crianças em ambientes de cuidado, promovendo momentos de aprendizagem, no qual sejam partícipes, e junto à família, possam incorporar saberes práticos mediados por saberes científicos. Diante das reflexões aqui iniciadas, o presente estudo teve como objetivo apresentar as experiências e as atividades vivenciadas por acadêmicos de Enfermagem durante um estágio curricular em Pediatria na orientação sobre higiene corporal voltado para crianças hospitalizadas e seus cuidadores.

\section{Descrição da experiência}

Trata-se de um relato de experiência que descreve aspectos vivenciados pelos autores, na oportunidade de um estágio supervisionado do componente curricular "Assistência de Enfermagem em Pediatria", num hospital de referência em trauma de Ananindeua, Estado do Pará, no período de setembro a novembro de 2015, após a prévia autorização da coordenação e direção. Trata-se de um olhar qualitativo, que abordou a problemática delineada a partir de métodos descritivos, observacionais e participativos a luz da problematização.

O relato de experiência é uma ferramenta da pesquisa descritiva que apresenta uma reflexão sobre uma ação ou um conjunto de ações que abordam uma situação vivenciada no âmbito profissional de interesse da comunidade científica 5 .

O projeto desta pesquisa não foi submetido ao Comitê de Ética em Pesquisa por se tratar de relato de experiência dos autores, com anuência do local onde ocorreu o estágio curricular obrigatório e garantias de confidencialidade dos dados. Utilizou-se das seguintes técnicas de coleta de dados: diário de campo, observação estruturada (pesquisador participante) e participação nas atividades (ação educativa). Não foram utilizados dados pessoais, apenas aqueles de interesse para o estudo, porém sem fazer qualquer menção a dados que pudessem identificar os participantes.

A problematização tem como eixo básico a ação-reflexão-ação que conduz o processo e tem nos estudos de Paulo Freire, a sua origem, quando o professor permite ao aluno se perceber como ser inserido no mundo tentando responder a novos desafios. Os problemas que devem ser estudados partem de um cenário real e têm seu trabalho político-pedagógico marcado por uma postura crítica da educação ${ }^{6}$. É descrita por um arco, como pode ser observado na Figura 1, no qual constam cinco etapas: observação do problema, identificação dos fatores mais importantes acerca do 


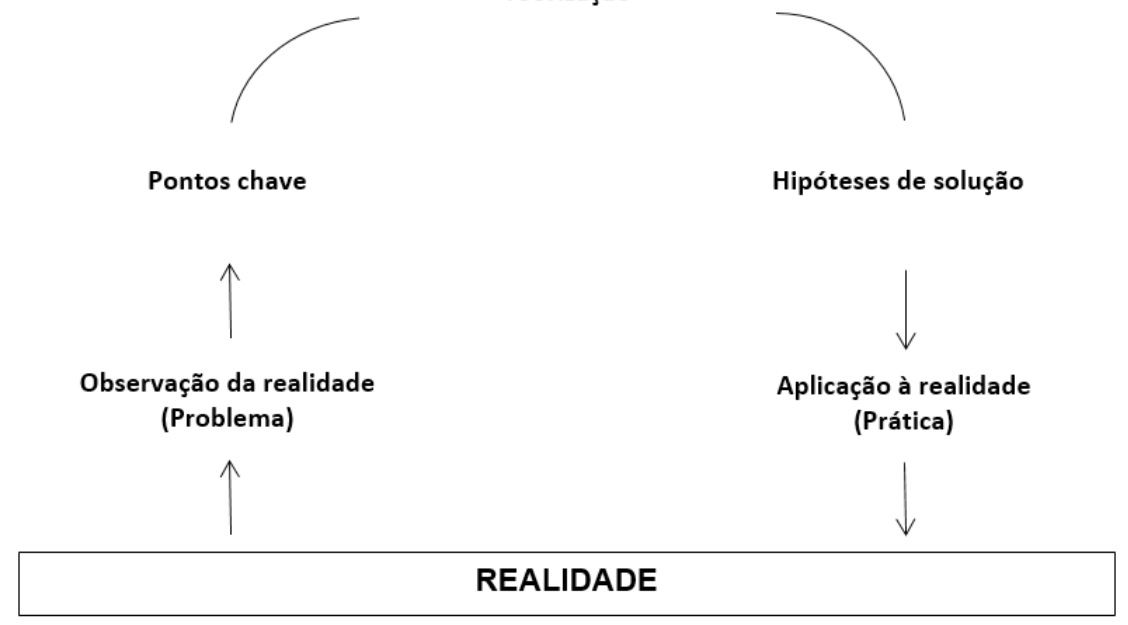

Figura 1. Método do Arco de Charles Maguerez.

problema, estudo das causas do problema, desenvolvimento de hipóteses de solução e, por fim, ações para resolver o problema ${ }^{8}$.

A problematização, portanto, foi a metodologia selecionada para o planejamento das atividades propostas nesta experiência. Durante a fase de observação da realidade, os discentes foram instigados pelo professor, partindo de um tema gerador "Hábitos saudáveis e qualidade de vida", a identificarem as necessidades das crianças hospitalizadas e de seus cuidadores com vistas a desenvolver uma ação educativa em saúde ao final do estágio.

Observamos que era frequente que as crianças e cuidadores apresentavam hábitos de higiene inadequados, como não tomar banho, não lavar as mãos e não escovar os dentes, por exemplo. Por isso, o tema escolhido para a ação educativa foi "higiene corporal", com pontos-chave na higienização das mãos, corte e limpeza das unhas, banho e escovação dental.

Em seguida, os discentes seguiram para a teorização, tendo como objetivo o levantamento de informações científicas em bases de dados da área da saúde, com o intuito de estabelecer a base teórico-científica e atualização de informações, de forma a subsidiar o planejamento da ação educativa.

A hipótese de solução gerada, então, foi a de elaborar uma ação de Educação em Saúde pautada nas necessidades de desenvolvimento e adequação de hábitos de higiene da população-alvo, com a preocupação de concretização e adoção desses hábitos não apenas no ambiente hospitalar, mas na vida cotidiana.

A ação educativa consistiu-se de uma peça teatral. Por ser voltada para o público infantil, optou-se por uma atividade lúdica, que facilita a aproximação da equipe com as crianças, além de promover a socialização e facilitar o acolhimento das crianças e seus familiares que, durante a internação hospitalar, encontram-se em um momento de fragilidade física e emocional. É descrito que a dramatização é uma ferramenta valiosa para o desenvolvimento de práticas educativas voltadas para crianças, pois faz parte do dia-a-dia da criança, principalmente durante suas brincadeiras, além de agregar outra ferramenta muito apreciada pelas crianças: as histórias ${ }^{9}$.

Após a criação do roteiro da peça, figurino e cenário, surgiu o desejo de transformar a apresentação de uma dramatização em algo mais atraente e significativo para as crianças e seus cuidadores. A equipe assistencial da clínica foi consultada a respeito da possibilidade de oferta de lanches e brindes durante a ação educativa. Os discentes angariaram fundos e doações para a criação de kits de higiene para presentear as crianças e seus cuidadores; elaboraram algumas brincadeiras, e ao final realizaram uma roda de conversa para a socialização e feedback. 
O desenvolvimento da atividade ocorreu em um único dia, no período da tarde e no espaço da própria clínica pediátrica (hall de acesso). Participaram todas as crianças internadas (dezenove) e seus respectivos cuidadores, totalizando 38 participantes. O perfil da clínica pediátrica dessa instituição é constituído por crianças na faixa etária de 1 mês a 12 anos de idade, vítimas de lesões por causas externas (traumas).

A atividade iniciou-se com a brincadeira de "quebra-gelo" com balões, da qual todas as crianças puderam participar, com o objetivo de socialização e apresentação das crianças e demais participantes da atividade. Em seguida, foi apresentada a dramatização, cujo roteiro apresentava uma criança com hábitos precários de higiene, demonstrando os problemas em potencial que a falta de higiene podem trazer a saúde das pessoas. No desenrolar da história, a personagem desenvolvia hábitos saudáveis de higiene corporal e melhorava a sua qualidade de vida, inclusive conquistando novos amigos.

Assim, realizou-se uma roda de conversa ao final da peça teatral, na qual as crianças e seus familiares foram questionados acerca das lições apresentadas na história e sua opinião sobre o assunto abordado. Esta etapa da atividade teve grande importância no processo de Educação em Saúde, pois proporcionou o esclarecimento das dúvidas das crianças e de seus cuidadores, além de ter demonstrado a nítida aceitação e interesse por parte da população-alvo em relação ao tema.

É importante mencionar, ainda, que no dia do desenvolvimento da atividade outros acadêmicos (Psicologia e Terapia Ocupacional) estavam desenvolvendo estágio supervisionado na Clínica Pediátrica e contribuíram para a organização do cenário, transporte das crianças com mobilidade física prejudicada, distribuição dos lanches e brindes, entre outros.

Após a roda de conversa, foram distribuídos os brindes, que consistiram em kits de materiais para a prática da higiene corporal, com xampu, sabonete, escovinha para unhas, tesoura para cortar as unhas (este item foi entregue apenas aos cuidadores devido ao risco de utilização pelas crianças), escova dental e creme dental. As crianças e seus cuidadores foram orientados sobre a forma de usar esses materiais. A atividade foi encerrada com um lanche e socialização dos participantes.

\section{Resultados e impactos}

A experiência proporcionou a constatação da importância das ações de Educação em Saúde, que devem ser executadas nos mais diversos cenários, inclusive no ambiente hospitalar.

É pela importância dos saberes diferentes que se vêa importância do aperfeiçoamento do Enfermeiro, devendo-se pensar sempre no Art. 14 do código de ética dos profissionais de enfermagem, que refere que o profissional deve aprimorar os seus conhecimentos técnicos, científicos, éticos, culturais e humanísticos, em benefício da pessoa, família e coletividade e do desenvolvimento da profissão ${ }^{10}$.

A promoção da saúde é compreendida como combinação de apoios educacionais e ambientais que visam atingir ações e condições de vida conducentes à saúde e que envolvem a formação de atitudes e valores que levam os indivíduos ao comportamento autônomo, revertendo em benefício à sua saúde e à daqueles que estão à sua volta ${ }^{11}$.

O tema desta ação educativa foi selecionado a partir da identificação de um problema do cotidiano da população-alvo, o que permitiu que os acadêmicos compreendessem que as ações de Educação em Saúde são válidas apenas quando atendem às demandas, necessidades e expectativas da população.

Partindo dessa compreensão, enfatizamos que as ações de promoção da saúde não deveriam se limitar apenas a dar conhecimentos. Precisamos motivar o aprendizado, estimular o aluno a analisar e avaliar as fontes de informação e, com isso, torná-lo capaz de adotar práticas comportamentais com base no conhecimento ${ }^{11}$. 
O interessante dessa atividade foi que o tema abordado, em primeira análise, não chamaria a atenção dos participantes no contexto em que foi realizada.

Enfatiza-se a importância de realizar ações em saúde pautadas nos interesses e necessidades que emergem da realidade das pessoas. É provável que o impacto causado por uma ação previamente planejada, sem levar em consideração o interesse dos participantes, sem que os pesquisadores tivessem contato com a realidade da população-alvo e observassem as necessidades reais, fosse pouco aceita pelos envolvidos.

É descrito que, durante muito tempo, a educação em saúde centrou sua ação nas individualidades, tentando mudar comportamentos e atitudes sem, muitas vezes, considerar as inúmeras influências provenientes da realidade em que as pessoas estavam inseridas. Era comum acontecerem ações isoladas voltadas ao trabalho para saúde, partindo de uma visão assistencialista de educação e sem discutir a conscientização acerca do tema saúde e suas inter-relações para o equilíbrio dinâmico da vida ${ }^{12}$.

Consideramos que, se as ações em saúde fossem pautadas apenas nas expectativas dos discentes e docentes, poderiam resultar em uma ação cujo tema estivesse relacionado à patologias e descontextualizadas da realidade dos participantes, causando pouco impacto e tímidas mudanças de comportamentos. Entretanto, a vivência e contato prévio e dialógico com as crianças e seus cuidadores demonstrou que o problema que precisava de solução era outro, completamente diferente, e não estava relacionado a doenças.

O perfil das crianças e cuidadores, apesar de não terem sido coletados dados descritivos referentes à condição socioeconômica nesta experiência, geralmente é de baixa renda, a maioria procedente de municípios e distritos do interior do Estado e, frequentemente, relacionados à produção agrícola. Durante a roda de conversa foi observado que esses participantes não tinham acesso a ações de Educação em Saúde.

$\mathrm{O}$ feedback proporcionado pelos participantes da atividade aos organizadores foi bastante positivo, com aceitação e valorização do conhecimento construído, com profundas reflexões identificadas por meio dos discursos. Assim, constatou-se que uma atividade de Educação em Saúde, pautada nas reais necessidades da população-alvo, pode provocar mudanças significativas nos hábitos de vida, promovendo a saúde. Ressalta-se que a orientação e planejamento da atividade a partir de um problema real identificado na experiência prática no campo de estágio foram fundamentais para subsidiar a ação.

Além desse feedback proporcionado no dia da atividade, os organizadores mantiveram contato com a equipe assistencial da Clínica Pediátrica e foi relatado por esta que as crianças e seus cuidadores estavam apresentando diferentes comportamentos em relação à sua higiene corporal, o que demonstrou o impacto positivo da ação desenvolvida.

Assim, constatamos que uma atividade de Educação em Saúde, pautada nas reais necessidades da população-alvo, pode provocar mudanças significativas nos hábitos de vida, promovendo a saúde. Ressaltamos ainda que, a orientação e planejamento da atividade a partir de um problema real identificado na experiência prática no campo de estágio supervisionado foram facilitados pela metodologia empregada, a partir da problematização.

A ação do profissional da saúde nas praticas educativa requer uma assistência fundamentada no domínio teórico-prático, construídos a partir de vivencias práticas e durante a graduação. Observamos também a importância das reuniões de apoio e orientação, sendo que é necessário um vinculo de confiança entre educador e o educando juntamente com a família. Assim a promoção de ações que visem à melhoria da qualidade de vida tende a ser facilitada.

Enfatizamos que não é de hoje que se reconhece o vínculo entre a saúde e a educação. Sob o argumento desta íntima ligação entre as duas áreas, existe ao menos um 
consenso: bons níveis de educação estão relacionados a uma população mais saudável assim como uma população saudável tem maiores possibilidades de apoderar-se de conhecimentos da educação formal e informal ${ }^{13}$.

\section{Considerações finais}

O desenvolvimento da ação Educativa em Saúde no ambiente hospitalar, com a temática "hábitos saudáveis de higiene corporal" produziu impacto positivo no comportamento de crianças hospitalizadas e de seus cuidadores. A forma como a informação sobre o tema foi abordado possibilitou a participação de todos de forma ativa facilitando o aprendizado.

A vivencia proporcionou profundas reflexões em relação à importância da Educação em Saúde e do planejamento sistemático de atividades educativas que atendam às necessidades reais da população, o que foi facilitado nesta experiência pela escolha da estratégia metodológica, problematização.

Esta experiência proporcionou ainda a oportunidade de desenvolver habilidades nos docentes como facilitadores do processo educativo, no sentido de promover mudanças e garantir um contexto de vida saudável para a comunidade. Para tanto há a necessidade de planejamento, organização e sensibilização da equipe e usuários para a participação das atividades propostas.

Por meio desta ação, foi proporcionado um aprendizado teórico-prático de etapas como a identificação das necessidades, possibilidade da realização da atividade, integração ensino-serviço e avaliação das atividades desenvolvidas. Entendemos que a educação em saúde deve contribuir para a auto-formação do indivíduo, de modo a ensinar e promover a qualidade de vida e condição humana, ensinar a viver e ensinar como se tornar cidadão, além de ser compreendida como uma proposta a fim de desenvolver no indivíduo e no grupo a capacidade de avaliar de forma crítica a sua realidade, como também, de decidir acerca de ações conjuntas para resolver problemas no seu microespaço e modificar os condicionantes de saúde-doença, de modo a organizar e realizar a ação e de avaliá-la com espírito crítico.

\section{Referências}

1. Remor CB, Pedro VL, Ojeda BS, Gerhardt LM. Percepções e conhecimentos das mães em relação às Práticas de higiene de seus filhos. Esc Anna Nery. 2009;13(4):786-92. http://dx.doi.org/10.1590/S1414-81452009000400014.

2. Melo EMOP, Ferreira PL, Lima RAG, Mello DF. The involvement of parents in the healthcare provided to hospitalzed children. Rev Lat Am Enfermagem. 2014;22(3):432-9. http://dx.doi.org/10.1590/0104-1169.3308.2434.

3. Silveira A, Neves ET, Zamberlan KC, Pereira FP, Arrué AM, Pieszak GM. A família de crianças/adolescentes hospitalizados: o grupo como estratégia de cuidado. Ciência. Cuid e Saúde [Internet]. 2012 [citado em 2016 Dez 16];11(2):402-7. Disponível em: http://www.periodicos.uem.br/ojs/index.php/CiencCuidSaude/article/view/19613/pdf

4. Pennafort VPS, Silva ANS, Queiroz MVO. Percepções de enfermeiras acerca da prática educativa no cuidado hospitalar a crianças com diabetes. Rev Gaucha Enferm. 2014;35(3):130-6. http://dx.doi.org/10.1590/1983-1447.2014.03.43313.

5. Fernandes NC, Teixeira PRA, Sá AMM, Medeiros LM, Peixoto IVP. Monitoria acadêmica e o cuidado da pessoa com estomia: relato de experiência. Rev Min Enferm [Internet]. 2015[citado em 2016 Dez 16];19(2):238-41. Disponível em: http://www.reme.org.br/artigo/detalhes/1018

6. Xavier LN, Oliveira GL, Gomes AA, Machado MFAS, Eloia SMC. Analisando as metodologias ativas na formação dos profissionais de saúde: uma revisão integrativa. Sanare [Internet]. 2014 [citado em 2016 Dez 16];13(1):76-83. Disponível em: https://sanare.emnuvens.com.br/sanare/article/view/436/291

7. Mitre SM, Siqueira-Batista R, Girardi-de-Mendonça JM, et al. Metodologias ativas de ensino-aprendizagem na formação profissional em saúde: debates atuais. Cien Saude Colet. 2008;13(2, Supl 2):2133-44. PMid:19039397.

8. Berbel NAN. A problematização e a aprendizagem baseada em problemas: diferentes termos ou diferentes caminhos? Interface Comunicacao Saude Educ. 1998;2(2):139-54. http://dx.doi.org/10.1590/S1414-32831998000100008.

9. Sant'Anna VLL, Souza EM, Cruz LG, Silva MR. As práticas educativas vivenciadas pelo pedagogo nos hospitais: possibilidades e desafios. Pedagog. Ação [Internet]. 2010 [citado em 2016 Dez 16];2(1):1-103. Disponível em: http://200.229.32.55/index. $\mathrm{php} /$ pedagogiacao/article/viewFile/4480/4605

10. Ribeiro VCS, Nogueira DL, Assunção RS, Silva FMR, Quadros KAN. Papel do enfermeiro da estratégia de saúde da família na prevenção da gravidez na adolescência. R. Enferm. Cent. O. Min [Internet]. 2016 [citado em 2016 Dez 16];1(6):1957-75. Disponível em: http://www.seer.ufsj.edu.br/index.php/recom/article/viewFile/881/1006 


\section{iihe}

11. Ilha PV, Lima APS, Rossi DS, Soares FAA. Intervenções no ambiente escolar utilizando a promoção da saúde como ferramenta para a melhoria do ensino. Revista Ensaio [Internet]. 2014 [citado em 2016 Dez 16];16(3):35-53. Disponível em: http:// www.scielo.br/pdf/epec/v16n3/1983-2117-epec-16-03-00035.pdf

12. Gonçalves FD, Catrib AMF, Vieira NFC, Vieira LJES. A promoção da saúde na educação infantil. Interface Comunicacao Saude Educ. 2008;12(24):181-92. http://dx.doi.org/10.1590/S1414-32832008000100014.

13. Casemiro JP, Fonseca ABC, Secco FVM. Promover saúde na escola: reflexões a partir de uma revisão sobre saúde escolar na América Latina. Cien Saude Colet. 2014;19(3):829-40. PMid:24714896. http://dx.doi.org/10.1590/1413-81232014193.00442013.

\section{Contribuição dos autores}

Cinthya Lorena Bezerra Sarmanho, Kéven Lorena de Paula Gonçalves, Maicon de Araujo Nogueira, Michele de Freitas Melo participaram de todas as etapas da pesquisa e da confecção do manuscrito. Renato da Costa Teixeira participou na concepção inicial, acompanhamento, orientação do estudo e elaboração e revisão crítica do manuscrito. 